\title{
Eating Disorders Risk Profiles in the Adolescents in the Abruzzo's Region in Italy: a Cross-Sectional Survey in the Aftermath of an Earthquake
}

\author{
Francesco Masedu ${ }^{1 *}$, Annamaria Cappa Monti ${ }^{2}$, Mario Di Pietro ${ }^{3}$, Luigi Gileno ${ }^{4}$, Marco Valenti $^{5}$, Maria
} Chiara Pino $^{6}$, Melania Mariano ${ }^{7}$, Mazza Monica $^{8}$

${ }^{1}$ Prof., Section of Environmental Medicine and Clinical Epidemiology, Department of Applied Clinical Sciences and Biotechnology, University of l'Aquila,

L'Aquila, Italy.

${ }^{2}$ President UNICEF, Delegation of the Abruzzo's Region, Italy.

${ }^{3}$ Director of the Unity of Neonatology and Pediatry, Atri Hospital, Italy.

${ }^{4}$ Psychotherapist UNICEF, Delegation of the Abruzzo's Region, Italy

${ }^{5}$ Head of the Section of Environmental Medicine and Clinical Epidemiology, Department of Applied Clinical Sciences and Biotechnology, University of l'Aquila,

L'Aquila, Italy

${ }^{6} \mathrm{PhD}$ student, Health and Environmental Sciences, Department of Clinical Medicine, University of l'Aquila, L'Aquila, Italy

${ }^{7} \mathrm{PhD}$ student, Section of Psychology, Department of Applied Clinical Sciences and Biotechnology, University of l'Aquila, L'Aquila, Italy

${ }^{8}$ Prof., Section of Psychology, Department of Applied Clinical Sciences and Biotechnology, University of l'Aquila, L'Aquila, Italy.

Received: September 17, 2016; Accepted: October 05, 2016; Published: October 22, 2016

*Corresponding author: Francesco Masedu, PhD, Assistant Professor of Biostatistics and Epidemiology, Department of Applied Clinical Sciences and Biotechnology, University of L'Aquila, Italy, Via Vetoio, Coppito 2, L'AQUILA, ITALY, Tel: +39 0862433736 ; Fax: +39 0862 432236;E-mail:

francesco.masedu@univaq.it

\section{Abstract \\ Background: In the Abruzzo's Region on the 6 April 2009 occurred a catastrophic earthquake, which divided the population into a natural cohort of subjects exposed to the event in the internal area of the territory and a not exposed fraction of the population in the coastal area. The present cross-sectional research has been devoted to get a general profile of the adolescents (13-14 years old) risk of eating disorders. These issues are of interest especially with known eating disorders like anorexia nervosa, bulimia and binge eating syndrome.}

Methods: The analysis has been carried out using the EDI-3 questionnaire' scales on the risk of eating disorders behaviours (EDBR), administered by specifically trained psychologists. The sample included 434 school students with an age of 13/14 years old ( $50.46 \%$ boys, $49.54 \%$ girls). Data have been analysed using a twoway, fixed effects, MANOVA design, comparing the adolescents' profiles between boys and girls exposed and not exposed to the earthquake. A principal component analysis (PCA) provided an importance ordering of the EDI-3 scales between boys and girls. Statistically significant differences have been detected between eating risk profiles.

The study highlighted important aspects in terms of Public Health management and decision making. Some indicators coming from the psychological scales suggest a propensity to specific forms of psychological risk and eating behaviour higher in the inland areas. Practical implications of the survey conducted are the statistical evidence of two structured cluster of psychological risk profile associated with EDBR and the concurrent validity with previous longitudinal studies.

Keywords: Earthquake; Adolescents; Behavioural Eating Disorder Risk; EDI-3 Questionnaire; Risk Profile-Plot;

\section{Introduction}

In the Abruzzo's Region on the 6 April 2009 occurred a catastrophic earthquake which structured a natural cohort of subjects exposed to the event in the internal area of the territory and a not exposed fraction of the population in the coastal area. This region of Italy was therefore divided into a coastal area and a seismic area, commonly called "seismic crater". Teenagers experienced the same event with consequences coming from the interactions of specific aspects of their psychological development, age as well as the social and human context they lived in. This situation resulted, as documented by numerous studies $[1,2,3]$, into a territorial division in terms of Quality of Life (QOL).

The present survey, promoted by the UNICEF delegation of the Abruzzo's Region, conducted a comparison and prevalence estimations of the magnitudes of risk eating disorders behaviours (EDBR) in the Region between the cohort of the adolescents exposed (Seismic Crater SC) and not exposed (Coastal Area CA) after more than 5 years after the earthquake. This interest stems from the awareness of the importance of eating behaviours in the adolescence and its related importance in this vulnerable age for the identification of psychological critical aspects [4].

The psychiatric classification for many years' counts among its diagnostic categories the eating disorders, especially the children and the young people, but it shows a special interest in the last twenty years [5] with particular reference to Anorexia 
Nervosa (AN) and Bulimia Nervosa (BN). The objective dangers as well as the combined personal psychological distress framework undermine the QOL of those involved especially if associated with other severe psychiatric comorbities [6]. These disorders were until fairly recently considered the preserve of women [7], but the number of males, particularly in adolescence is growing $[6,7]$. Studies of the early 90 s already indicated that AN as the third chronic disease most common among girls aged 15 to 19 years [8]. It is also associated with severe medical complications and its mortality rate exceeds the expected incidence of death from all causes in women between 15 and 24 years $[9,10]$. BN shows lower mortality rates $[11,12,13]$ indubitably of Public Health interest for the teenagers [14].

The Italian Ministry of Health [15] documents how even in Italy the EDBR are taking an increasing interest on the basis of epidemiological indications. Epidemiological surveys estimate in Western countries in women aged between 12 and 25 years the prevalence of anorexia nervosa which varies from 0.2 to $0.8 \%$ for bulimia nervosa a prevalence of around $3 \%$, and EDBR nonspecific 3.7 and $6.4 \%$. The literature documents an age of onset between 10 and 30 years with a mean of 17 years, with a tendency to increase especially for Bulimia and Binge Eating Disorders (BED) [16]. The literature on EDBR in the Italian population is affected by problems of quantity, quality and comparability. In terms of quantity there are few studies concerning the Italian population, a problem which is aggravated if the focus on the population of adolescents is requested. This issue is worth emphasizing not just about the 'Italy but the entire European continent [17]. The quality is affected by a strong heterogeneity in the reference diagnostic classifications, in the testing methods used and the epidemiological pattern. So that their usability is limited by generalizability and comparability issues.

The review of Steinhausen and Weber which addressed the BN, using a sample of 5653 subjects, makes clear differences in the design and the diagnostic criteria exploited, emphasizing the necessary cautions in the interpretation of the results [18]. Comparability is a methodologically complex issue also because the estimates describing the EDBR are derived from extremely heterogeneous health care systems (Japan, USA, Germany, Norway, Finland, and Sweden).

Some research suggest a very chaotic trend structure of the EDBR worldwide although for the reasons stated for this increase is far from being supported by sufficient scientific evidence [19]. In terms of quality is sufficient to say the scarcity of longitudinal studies and clinical bias in the recruitment of subjects.

The indications of the prevalence and incidence resulting from the international literature of recent years appears sometimes with discordant results presumably influenced by the socio-cultural context as well as the diagnostic approach. Then we observe a strong dependence on the socio-cultural context $[19,20]$.

The estimated prevalence of in European countries (Belgium, France, Germany, Italy, Netherlands, Spain) is $0.93 \%$ higher in women older than or equal to 18 years [21]. The overall prevalence of EDBR in Italy [21] is equal to $3.3 \%$ of subjects with age greater or equal to 18 years. Also with regard to Italy the rate of incidence of hospitalization for AN in patients aged 10-19 years in Italy hospitalized for the first time in 2004-2005 and not hospitalized for AN between 2001 and 2003 amounted to 22.8 per 100,000 among women and 2.0 per 100,000 among men. The rate of incidence of hospitalization for AN in women aged 15-19 years admitted for the first time in 2004-2005 and not hospitalized for AN between 2001 and 2003 amounted to 31.7 per 100,000 [22].

The aim of the UNICEF study addressed the epidemiological boundaries of the spectrum of eating disorders in the Abruzzo's Region in a population between 13 and 14 years of age. The survey associated the psychological profiles of the EDI-3 scales, previously administered by qualified operators, and the exposition to the seism stratifying by gender.

\section{Methods}

\section{Survey design and questionnaire assessment EDBR}

The Eating Disorder Inventory EDI-3 provides a standardized clinical evaluation of the symptomatology associated with eating disorders [23]. EDI-3 is built on scales measuring constructs related to psychological domains deemed relevant in the aetiology, stability or variation of the key symptoms of patients with eating disorders [24].

The EDI-3 is composed of 91 items, with a graduated response from "Always" to "Never." The test is divided into 12 main stairs: 3 Eating Disorder Risk Scales (Drive For Thinness (DT), Bulimia (B), Body Dissatisfaction (BD)); 9 Psychological Scale (Low SelfEsteem (LSE), Personal Alienation (PA), Interpersonal Insecurity (II), Interpersonal Alienation (IA), Interoceptive Deficits (ID), Emotional Dysregulation (ED), Perfectionism (P), Asceticism (A ) Maturity Fears (MF)); 6 Psychological Composite Scores (Eating Disorder Risk Composite (EDRC), Ineffectiveness Composite (IC), Interpersonal Problems Composite (IPC), Affective Problems Composite (APC), Overcontrol Composite (OC), Global Psychological maladjustment Composite (GPMC)).

The choice of instrument was oriented by the specificity of the age (bigger of 13 years) for which it was designed, the ease of administration and its proven use from EDI [25] and subsequently the EDI-2 questionnaires in 1991.

The readings of the scores were conducted taking into account the T-scores of the EDI-3 scales, stratifying the sample estimates by gender and geographical areas. Such stratification reflects the need to account for territorial locations which, for the geography of the Region, mirror the administrative districts that, in a broader socio-cultural sense, result in different eating behaviours. This distinction was further characterized by the earthquake exposition of the 2009 which divided the population into the two cohorts of exposed and unexposed subjects. Many longitudinal researches maintain and support the existence of clusters of psychological and social discomfort, which let us permit to interpret the outcomes in terms of concurrent validity [26]. 


\section{Target population and sampling}

The reference population was that of boys and girls aged between 13 and 14 years. The survey was cross-sectional, carried out using a two sampling stages that considered land areas and schools, after the authorization from the school and legal tutors, by a team of trained psychologists who administered the EDI-3 questionnaires. The collected data was then sent for analysis to the bio statistical group. Data collection requested four months of work (from September 2014 to December 2014) by the operators.

The territories involved were those of the Provinces of L'Aquila, in the inland areas, mainly affected by the earthquake of 6 April 2009, and the Provinces of Pescara, Chieti and Teramo for the coastal areas, with a bigger anthropization. Demographic references for the calculation of the sampling fraction have had as a source ISTAT (National Institute of Statistics) data to January $1,2014$.

Budget limits and human resources constrained the sample size to 500 subjects Table 1 .

The sample included 434 school students with an age of 13/14 years old, divided into 219 males and 215 females, respectively, $50.46 \%$ and $49.54 \%$ of the total of subjects who completed the questionnaire.

The table indicates some recruitment issues in the area of Chieti and Pescara with a compliance rate of about $86.8 \%$ that in the case of L'Aquila and Teramo is negligibly less than $100 \%$.

\section{Statistical analysis}

The statistical analysis was conducted according to a twoway, fixed effect, MANOVA design. The primary outcome variable was constructed based on the vector containing the scores of eating disorder scales, psychological, and the composite scores of eating disorder risk of EDI-3 questionnaire. The factors used were the kind and the province of belonging boys.

The overall statistical significance was set with $\alpha=5 \%$. It was

Table 1: Abruzzo's region administrative districts students

\begin{tabular}{|c|c|c|c|c|c|}
\hline (Age 13-14 years) & L'AQUILA & PESCARA & CHIETI & TERAMO & Total \\
\hline Men & 1357 & 1575 & 1885 & 1429 & 6246 \\
\hline \% & 21.73 & 25.22 & 30.18 & 22.88 & 100 \\
\hline Sampling size & 60 & 51 & 51 & 57 & 219 \\
\hline Women & 1234 & 1402 & 1640 & 1368 & 5644 \\
\hline$\%$ & 21.86 & 24.84 & 29.06 & 24.24 & 100 \\
\hline Sampling size & 48 & 52 & 59 & 56 & 215 \\
\hline & & & & & \\
\hline Total & 2591 & 2977 & 3525 & 2797 & 11890 \\
\hline \% & 21.79 & 25.04 & 29.65 & 23.52 & 100 \\
\hline $\begin{array}{c}\text { Total sampling } \\
\text { size }\end{array}$ & 108 & 103 & 110 & 113 & 434 \\
\hline & *Source ISTAT 1st January 2014. & & \\
\hline
\end{tabular}

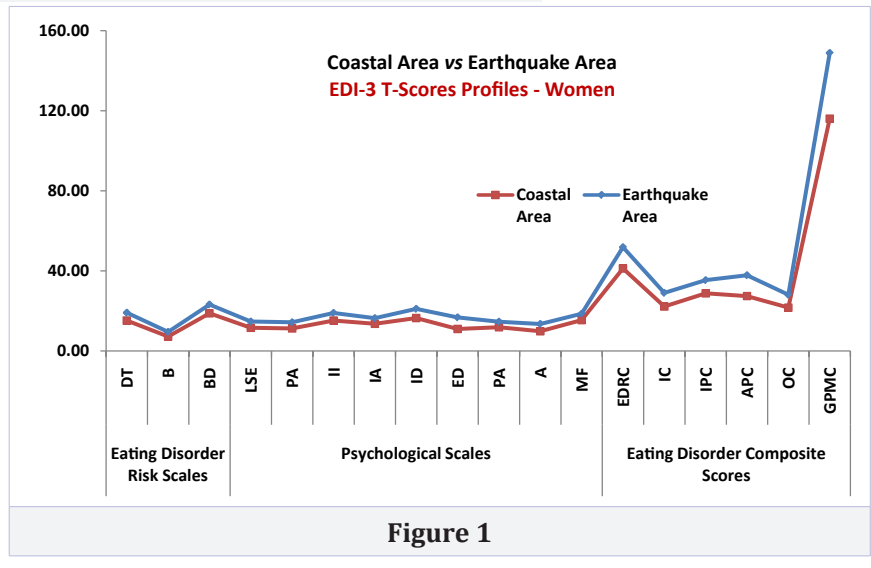

calculated the Cronbach's alpha for the items of the questionnaire (0.87). The assumptions of univariate and multivariate normality of the variables were tested using the Doornik-Hansen tests and Shapiro-Wilks for the univariate case. Statistical significance for multivariate analysis was adjusted for multiple testing in 0016 through the equation of Dunn-Sidak. The analysis of the sub scales has used linear regression models having as predictors gender and geographical location of the respondents. The comparison of the EDI-3 profiles according to the earthquake exposition, stratified by gender, was performed using the Hotelling test.

The overall assessment of the role of the individual EDI-3 scales has been dealt with an analysis of the dominant eigenvalues of the main components, so that the EDI-3 sub scales scores has been sorted in the groups of the boys and the girls, highlighting specific differences by gender in the risk of eating disorders.

Statistical analysis was carried out using the statistical software STATA (version 13).

\section{Results}

The research detected, on average, for the surveyed sample, according to gender and territorial distribution, typical T-scores (Table 2). However, some scales showed critical statistically significant differences between boys and girls resulted in epidemiological estimations of the scales' scores which should be considered in the well-being of the adolescents. The BMI does not show statistically significant differences ( $p>0.05)$. The boys and girls show BMI normal, compatible with those of their age with an average value of $19.43 \pm 3.1219 .78 \pm 3.55$ for males and for females. The estimated difference of DT (Stimulation of the thinness) was lower in boys than in girls with a reduction of $5.91 \pm 1.49$ points $(\mathrm{p}<0.000)$. The estimated prevalence of high DT scores is equal to $12.84 \%$ (9.85-16.35) \%. The estimated difference of Bulimia (B) by gender of children with an average reduction for males than females of 3.681 .20 points $(p<0.002)$ .The estimated prevalence in the population with high $B$ is equal to $5.73 \%$ (3.74-8:35) \%. Aspects such as Body dissatisfaction (BD) has a low prevalence estimate of $0.92 \%$ (0:25 to $2: 33) \%$. The scale IA of the Interpersonal Alienation, which assesses the estrangement and distrust of personal relations, reaches in the sample for the modality "high" 64.91\% (60.22-69.39) \%. The 
scale ID of the Interoceptive Deficits gets a sample percentage for the modality "high" of $26.38 \%$ (22.30-30.78) \%. The prevalence estimation obtained for the Emotional Dysregulation ED, for the modality "high", is 53.21\% (48.40-57.97) \% and for scale Maturity Fears (MF) we estimated 65.37\% (60.69-69.83) \% "high scores".

The overall evaluation score used the EDRC (of eating disorder risk): sum of the DT, B and BD scales. The statistical model showed statistically significant differences between the values of eating disorder risk (EDRC) by gender. The analysis estimated a reduction of EDRC $11.09 \pm 2.90$ points in the boys ( $p<0.000$ ) lower than in the girls of the same age, with a sample fraction prevalence of "high scores" equals to $14.22 \%$ (11.08-17.85). Affective Problems (APC) showed for the boys a reduction of the APC score of $5.53 \pm 2.53(p<0.029)$ than girls of the same age, with prevalence of "high scores" equals to $5.05 \%$ (3.19-7.54). The prevalence of those with a composite score of General Psychological Maladjustment (GPMC) "high" amounts to 93.12\% (90.32-95.31). The statistical model showed statistically significant differences between the values of the general psychological maladjustment (GPMC) according to gender. The analysis has estimated a reduction of the GPMC score for boys of $13.36 \pm 5.82(\mathrm{p}<0.022)$ than girls.

The result of the analysis of multivariate variance (MANOVA) indicates, in accordance with the above analysis, an overall dominance of the scores in the area of L'Aquila $\left(\Lambda_{\text {wILKS }}=0.279, \mathrm{~F}\right.$ $=15: 25, \mathrm{p}<0.00)$. The MANOVA shows a statistically significant effect also with respect to the gender factor $\left(\Lambda_{\text {wILKS }}=0.839, \mathrm{~F}=\right.$ $5: 48, p<0.00$ ), or the diversity of profiles among boys and girls under varying administrative districts.

The EDI-3 profile-plots showed higher overall risk profiles in the areas exposed to the earthquake of 6 April 2009 (T2 = 47 120, $\mathrm{F}(14,404)=5.655, \mathrm{p}<0.000)$. This EDBR pattern is confirmed adjusting the analysis by gender $(\mathrm{T} 2=47.13, \mathrm{~F}(14,194)=3.154$, $\mathrm{p}<0.002$ ) (Fig. 2).

In order to select within the psychological determinants of eating behaviour a hierarchy in the impact of each of them in structuring the average behaviour of the population of Abruzzo's adolescents was conducted an analysis of the principal components (PC) overall and stratified by gender. The dominant eigen values have provided a sorting which discriminates between boys and girls Table 3 .

Sorting by gender we remark the identical position to kind of BMI. The scale far more relevant is that relating GPMC (Global Psychological maladjustment) that, albeit with different weights, retains its position in both genders.

The comparison of the tables of dominant eigen values by gender shows, for instance, that among the BD girls before $\mathrm{B}$, in boys instead the ordering is reversed B precedes BD; Similarly, again by way of example, in the IA adolescents precedes ID and the ID teenagers precedes IA with a clear reversal of the hierarchical importance.

It's plausible that the ordering of the stairs is the screening of latent structures of specific maturation of two genders that have to balance the need to adapt to external models and the evolution of its own internal model, an autonomous expression and personal growth in their relationship with food, nutrition and social related [27].

\section{Discussion}

The last two decades research on risk factors of EDBR has had a remarkable development that has nevertheless suffered from imprecise use of terms in the various scientific publications and, as pointed out [28], the generic term "risk factor" has often been used to describe different types of risk factors. A discussion on risk factors for EDBR calls for clarification with respect to the development of the disorder model. In the past, risk and etiological factors for psychiatric disorders we have been proposed or from a specific theoretical perspective (eg biological, cognitive - behavioural, psychodynamic), or from an integrated perspective (bio-psychosocial model). In both cases, the structure is a model in which are defined a series of risk factors that may be relevant for the development of a disorder. As evidenced by

\begin{tabular}{|c|c|c|c|c|c|}
\hline \multirow[b]{3}{*}{ EDI-3 Scales } & & \multicolumn{4}{|c|}{ Gender } \\
\hline & & \multicolumn{2}{|c|}{ Men } & \multirow{2}{*}{\begin{tabular}{|c|} 
Women \\
$\begin{array}{c}\text { Earthquake } \\
\text { Area }\end{array}$ \\
\end{tabular}} & \multirow[b]{2}{*}{$\begin{array}{l}\text { Coastal } \\
\text { Area }\end{array}$} \\
\hline & & $\begin{array}{c}\text { Earthquake } \\
\text { Area }\end{array}$ & $\begin{array}{c}\text { Coastal } \\
\text { Area }\end{array}$ & & \\
\hline \multirow{3}{*}{$\begin{array}{c}\text { Eating } \\
\text { Disorder } \\
\text { Risk Scales }\end{array}$} & DT & $9.58 \pm 6.30$ & $10.52 \pm 7.26$ & $19.10 \pm 9.04$ & $15.20 \pm 8.81$ \\
\hline & B & $6.30 \pm 6.30$ & $5.88 \pm 5.91$ & $9.52 \pm 5.82$ & $7.20 \pm 7.03$ \\
\hline & BD & $20.93 \pm 6.03$ & $16.98 \pm 8.03$ & $23.19 \pm 5.53$ & $18.83 \pm 6.91$ \\
\hline \multirow{9}{*}{$\begin{array}{c}\text { Psychological } \\
\text { Scales }\end{array}$} & LSE & $14.15 \pm 3.34$ & $10.37 \pm 5.30$ & $14.69 \pm 4.20$ & $11.56 \pm 5.13$ \\
\hline & PA & $13.40 \pm 3.82$ & $10.52 \pm 5.43$ & $14.31 \pm 5.27$ & $11.24 \pm 5.40$ \\
\hline & II & $19.58 \pm 3.42$ & $15.07 \pm 7.01$ & $18.98 \pm 3.76$ & $15.19 \pm 7.00$ \\
\hline & IA & $16.82 \pm 3.59$ & $12.65 \pm 5.96$ & $16.38 \pm 4.02$ & $13.59 \pm 6.04$ \\
\hline & ID & $15.13 \pm 8.01$ & $12.47 \pm 7.54$ & $21.04 \pm 8.20$ & $16.43 \pm 8.51$ \\
\hline & ED & $12.58 \pm 8.27$ & $9.15 \pm 6.75$ & $16.77 \pm 8.20$ & $10.98 \pm 7.49$ \\
\hline & PA & $11.93 \pm 5.06$ & $10.75 \pm 5.76$ & $14.60 \pm 5.77$ & $11.81 \pm 5.23$ \\
\hline & A & $9.17 \pm 5.28$ & $8.31 \pm 5.91$ & $13.48 \pm 6.82$ & $9.84 \pm 6.49$ \\
\hline & MF & $16.07 \pm 6.32$ & $13.73 \pm 6.05$ & $18.58 \pm 6.79$ & $15.40 \pm 6.32$ \\
\hline \multirow{6}{*}{$\begin{array}{l}\text { Eating } \\
\text { Disorder } \\
\text { Composite } \\
\text { Scores }\end{array}$} & EDRC & $36.82 \pm 13.66$ & $33.38 \pm 15.20$ & $51.81 \pm 15.90$ & $41.23 \pm 17.31$ \\
\hline & IC & $27.55 \pm 5.79$ & $20.08 \pm 10.01$ & $29.00 \pm 7.91$ & $22.22 \pm 9.76$ \\
\hline & IPC & $36.40 \pm 5.42$ & $27.72 \pm 12.08$ & $35.35 \pm 6.22$ & $28.78 \pm 11.96$ \\
\hline & APC & $27.72 \pm 14.35$ & $21.46 \pm 12.24$ & $37.81 \pm 14.90$ & $27.41 \pm 14.43$ \\
\hline & OC & $21.10 \pm 8.38$ & $19.06 \pm 9.32$ & $28.08 \pm 11.13$ & $21.65 \pm 9.53$ \\
\hline & GPMC & $128.83 \pm 30.87$ & $102.54 \pm 36.23$ & $148.83 \pm 33.78$ & $116.04 \pm 39.40$ \\
\hline
\end{tabular}

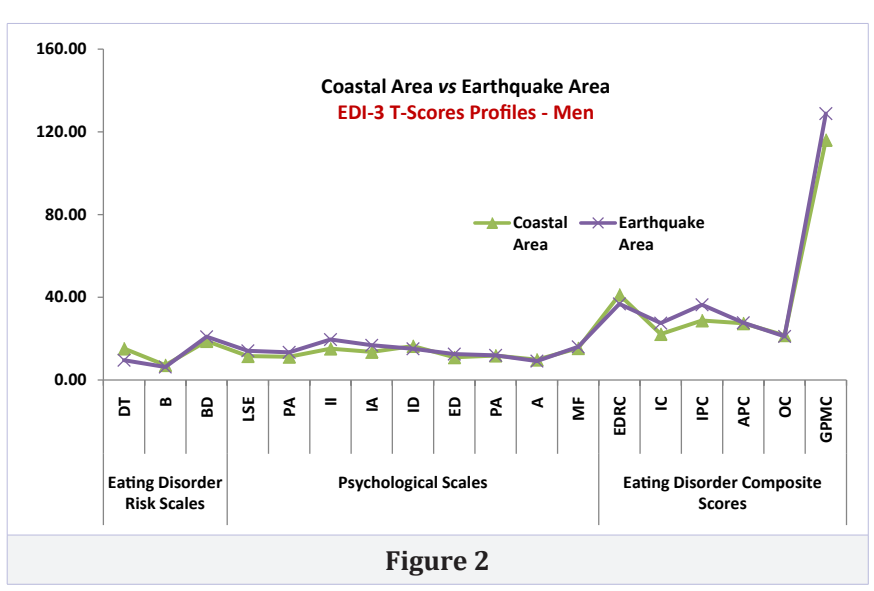


Table 3: EDI-3 sub-scales $\lambda$-Eigenvalues* Principal Component (PC) ordering in the Eating behaviour disorders

\begin{tabular}{|c|c|c|}
\hline EDI-3 Scales & Women PC $(\boldsymbol{\lambda}=\mathbf{9 . 9 1})$ & Men PC $(\boldsymbol{\lambda}=\mathbf{8 . 9 1})$ \\
\hline BMI & 0.020 & 0.005 \\
\hline P & 0.160 & 0.124 \\
\hline MF & 0.160 & 0.134 \\
\hline DT & 0.181 & 0.155 \\
\hline BD & 0.194 & 0.165 \\
\hline II & 0.198 & 0.190 \\
\hline IA & 0.202 & 0.198 \\
\hline B & 0.205 & 0.210 \\
\hline IPC & 0.219 & 0.216 \\
\hline ED & 0.223 & 0.217 \\
\hline LSE & 0.236 & 0.233 \\
\hline A & 0.236 & 0.242 \\
\hline OC & 0.243 & 0.256 \\
\hline ID & 0.246 & 0.257 \\
\hline EDRC & 0.249 & 0.259 \\
\hline PA & 0.254 & 0.262 \\
\hline APC & 0.258 & 0.264 \\
\hline IC & 0.271 & 0.278 \\
\hline GPMC & 0.311 & 0.329 \\
\hline
\end{tabular}

*The selected main component is only the first since it is the one most representative of the results obtained in the survey $(\sim 50 \%$ Total variability $\sim 50 \%$ )

a major systematic review [29], the perspective from which it is addressed the field of risk factors for EDB was that according to which, starting from a descriptive approach to evidence-based, it leads to the formulation of a more complex model that integrates the dynamic interactions between the risk factors. This type of approach by definition leads to overshadow a classification problem, that of the vicinity of a factor to the development of the disorder (for example predisposing factors vs. precipitating factors; factors or proximal vs. distal factors). These aspects are inserted into the model only after it was determined the status of a factor as a risk factor. The current challenge, which for now has not been able to give a definitive answer, is that it can integrate the risk factors within a model of development of the EDBR. The genesis and development of a pathogenetic model of EDBR will kept in mind two important classificatory distinctions: that between specific factors (i.e. the risk factors linked to the onset of EDBR) and factors (risk factors for the development of psychiatric disorders); that between the risk factors and protective factors.

The study highlighted aspects of obvious importance in terms of Public Health management and decision making in the Abruzzo's Region. Some indicators coming from the psychological scales suggest a propensity to specific forms of psychological risk and eating behaviour dominant in the inland areas. The crosssectional design of the survey is unsuitable to establish causal associations between the natural disaster and the EDBR, however, three aspects need to be appropriately emphasized to frame the practical implications of the survey conducted: the estimated prevalence values compared to the components of EDBR; statistical evidence of two structured cluster of psychological risk profile associated with EDBR; the concurrent validity with previous longitudinal studies carried out [30].

In Italy, there is no data of care activity on the benefits offered by services that are run by EDB. In this context, the monitoring of the assistance is very difficult and has entrusted exclusively to administrative data sources (SDO), and external evaluations of structural and organizational factors (e.g. institutional accreditation). It lacks a complete and unified quality of care for an insufficient spread between operators of assessment tools and monitoring of the health problems related to EDBR. It highlights the lack of studies of prevalence, incidence and mortality related to the EDBR in Italy on large non-clinical samples of adolescent's population in order to improve the quality and the amount of data which contribute to frame, if not to monitor, the EDBR from the epidemiological point of view.

It is unavoidable the necessity of standardized protocols for the epidemiological design so that can be adopted consistent and shared diagnostic tools for the mental disorders assessment (International diagnostic criteria of the DSM-V) to ensure adequate accuracy and reproducibility levels.

\section{Acknowledgements}

Authors would like to acknowledge and thank UNICEF for its organizational and administrative support.

\section{References}

1. Masedu F, Mazza M, Di Giovanni C, Calvarese A, Tiberti S, Sconci V, et al. Facebook, quality of life, and mental health outcomes in postdisaster urban environments: the l'Aquila earthquake experience. Front Public Health. 2014;22(2):286. doi:10.3389/fpubh.2014.00286.

2. Valenti M, Fujii S, Kato H, Masedu F, Tiberti S, Sconci V. Validation of the Italian version of the Screening Questionnaire for Disaster Mental Health (SQD) in a post-earthquake urban environment. Ann Ist Super Sanita. 2013;49(1):79-85. DOI:10.4415/ANN_13_01_13.

3. Mazza M, Pacitti F, Pino MC, Peretti S, Mazzarelli E. Investigation on quality of life and psychological well-being of citizens of L'Aquila after earthquake on April 6, 2009. Riv Psichiatr. 2014;49(3):145-51. doi:10.1708/1551.16913.

4. Berkowitz SA, Witt AA, Gillberg C, Råstam M, Wentz E, Lowe MR. Childhood body mass index in adolescent-onset anorexia nervosa. Int J Eat Disord. 2016. doi:10.1002/eat.22584.

5. Crow SJ, Peterson CB, Swanson SA, Raymond NC, Specker S, Eckert $\mathrm{ED}$, et al. Increased mortality in bulimia nervosa and other eating disorders. Am J Psychiatry. 2009;166(12):1342-6. doi:10.1176/appi. ajp.2009.09020247.

6. Mason TB, Lavender JM, Wonderlich SA, Crosby RD, Joiner TE, Mitchell $\mathrm{JE}$, et al. The role of interpersonal personality traits and reassurance seeking in eating disorder symptoms and depressive symptoms among women with bulimia nervosa. Compr Psychiatry. 2016;68:165171. doi:10.1016/j.comppsych.2016.04.013.

7. Byrne ME, Eichen DM, Fitzsimmons-Craft EE, Taylor CB4, Wilfley DE. Perfectionism, emotion dysregulation, and affective disturbance in relation to clinical impairment in college-age women at high risk for or 
with eating disorders. x Eat Behav. 2016;23:131-136. doi:10.1016/j. eatbeh.2016.09.004.

8. Pearson CM, Pisetsky EM, Goldschmidt AB, Lavender JM, Wonderlich SA, Crosby RD, et al. Personality psychopathology differentiates risky behaviors among women with bulimia nervosa. Int J Eat Disord. 2016;49(7):681-688. doi:10.1002/eat.22570.

9. Lucas AR, Beard CM, O'Fallon WM, Kurland LT. 50-year trends in the incidence of anorexia nervosa in Rochester, Minn.: a population-based study. Am J Psychiatry. 1991;148(7):917-922.

10. Sharp CW, Freeman CP. The medical complications of anorexia nervosa. Br J Psychiatry. 1993;162:452-462.

11. Birmingham CL, Su J, Hlynsky JA, Goldner EM, Gao M. The mortality rate from anorexia nervosa. Int J Eat Disord. 2005 Sep;38(2):143-146.

12. Keel PK, Mitchell JE, Davis TL, Crow SJ. Long-term impact of treatment in women diagnosed with bulimia nervosa. Int J Eat Disord. 2002;31(2):151-158.

13. Nielsen S. Standardized mortality ratio in bulimia nervosa. Arch Gen Psychiatry. 2003;60(8):851.

14. Keski-Rahkonen A, Mustelin L. Epidemiology of eating disorders in Europe: prevalence, incidence, comorbidity, course, consequences, and risk factors. Curr Opin Psychiatry. 2016 Nov;29(6):340-5. doi: 10.1097/YCO.0000000000000278.

15. Available at: http://www.salute.gov.it/portale/documentazione/

16. Micali N, Horton NJ, Crosby RD, Swanson SA, Sonneville KR, Solmi F, et al. Eating disorder behaviours amongst adolescents: investigating classification, persistence and prospective associations with adverse outcomes using latent class models. Eur Child Adolesc Psychiatry. 2016. DOI:10.1007/s00787-016-0877-7

17. Consensus Conference on Eating Disorders in adolescents and young adults. Edited by: De Virgilio G, Coclite D, Napoletano A, Barbina D, Dalla Ragione L, Spera G, Di Fiandra T. ISS (National Institute of Health) in Italian. Rapporti ISTISAN 13/6, 2013.

18. Steinhausen HC, Weber S. The outcome of bulimia nervosa: findings from one quarter century of research. Am J Psychiatry. 2009;166(12):1331-1341. doi: 10.1176/appi.ajp.2009.09040582

19. Pike KM, Hoek HW, Dunne PE. Cultural trends and eating disorders. Curr Opin Psychiatry. 2014;27(6):436-442. doi: 10.1097/ YCO.0000000000000100.

20. Hay PP, Bacaltchuk J, Stefano S, Kashyap P. Psychological treatments for bulimia nervosa and binging. Cochrane Database Syst Rev. 2009;7(4):CD000562. doi: 10.1002/14651858.CD000562.pub3.

21. Preti A, Girolamo Gd, Vilagut G, Alonso J, Graaf Rd, Bruffaerts R, et al. The epidemiology of eating disorders in six European countries: Results of the ESEMeD-WMH project. J Psychiatr Res. 2009;43(14):1125-1132. doi: 10.1016/j.jpsychires.2009.04.003

22. Gigantesco A, Masocco M, Picardi A, Lega I, Conti S, Vichi M. Hospitalization for anorexia nervosa in Italy. Riv Psichiatr. 2010;45(3):154-162.

23. Garner DM. EDI-3. Eating Disorder Inventory - 3. Italian version: Giannini M, Pannocchia L, Dalle Grave R, Muratori F, Viglione V. Giunti OS. Florence. 2013.

24.Segura-García C, Aloi M, Rania M, Ciambrone P, Palmieri A, Pugliese $\mathrm{V}$, et al. Ability of EDI-2 and EDI-3 to correctly identify patients and subjects at risk for eating disorders. Eat Behav. 2015;19:20-23. doi: 10.1016/j.eatbeh.2015.06.010.

25. Garner DM, Marion P. Polivy O, Polivy J. Development and validation of a multidimensional eating disorder inventory for anorexia nervosa and bulimia. Int J Eat Disord. 1983;2(2):15-34. Version of Record online: 13 FEB 2006|DOI:10.1002/1098-108X(198321)2:2<15::AIDEAT2260020203>3.0.CO;2-6.

26. Stratta P, Rossetti MC, di Michele V, Rossi A. [Effects on health of the L'Aquila (Central Italy) 2009 earthquake]. Epidemiol Prev. 2016;40(2 Suppl 1):22-31. doi: 10.19191/EP16.2S1.P022.044.

27. Dakanalis A, Carrà G, Calogero R, Fida R, Clerici M, Zanetti MA, et al. The developmental effects of media-ideal internalization and selfobjectification processes on adolescents' negative body-feelings, dietary restraint, and binge eating. Eur Child Adolesc Psychiatry. 2015;24(8):997-1010. doi: 10.1007/s00787-014-0649-1

28. Kraemer HC, Kazdin AE, Offord DR, Kessler RC, Jensen PS, Kupfer DJ. Coming to terms with the terms of risk. Arch Gen Psychiatry. 1997;54(4):337-343.

29. Jacobi C, Hayward C, de Zwaan M, Kraemer HC, Agras WS. Coming to terms with risk factors for eating disorders: application of risk terminology and suggestions for a general taxonomy. Psychol Bull. 2004;130(1):19-65.

30.V Watson HJ, Joyce T, French E, Willan V, Kane RT, Tanner-Smith EE, et al. Prevention of eating disorders: A systematic review of randomized, controlled trials. Int J Eat Disord. 2016;49(9):833-862. doi: 10.1002/ eat.22577. 